\title{
Una experiencia de aprendizaje combinado en Estadística para estudiantes de Psicología usando la evaluación como herramienta de aprendizaje
}

\section{A Blended Learning Experience in Statistics for Psychology Students Using the Evaluation as a Learning Tool}

Alberto Valentín Centeno, M. ${ }^{a}$ del Mar González-Tablas Sastre, M. ${ }^{a}$ Estrella Estrella López Pérez, Pedro Manuel Mateos García

Departamento Psicología Básica, Psicobiología y Metodología, Facultad de Psicología, Universidad de Salamanca, España. \{avc, marola, practpsi,pmateos\}@usal.es

\section{Resumen}

\begin{abstract}
La enseñanza de la asignatura de Estadística Aplicada a la Psicología, se ha basadoen diferentes modelos didácticos que incorporan metodologías activas de enseñanza. En esta experiencia se han combinado enfoques que priorizan el uso de las TIC con otros donde la evaluación se convierte en un elemento de aprendizaje. Ello ha supuesto la utilización de plataformas de apoyo virtual a la docencia que posibilitan un aprendizaje donde se combinan actividades presenciales y no presenciales. El diseño de los componentes del curso está inspirado en las dimensiones del modelo propuesto por Carless (2003). Este modelo utiliza la evaluación como elemento de aprendizaje. El desarrollo de esta experiencia ha mostrado cómo la propuesta didáctica ha sido interpretada positivamente por los estudiantes. Los alumnos reconocieron que tenían que aprender y comprender en profundidad los conceptos básicos de la asignatura, para que ellos puedan enseñar y evaluar a sus compañeros.
\end{abstract}

\section{Abstract}

Teaching statistics course Applied Psychology, was based on different teaching models that incorporate active teaching methodologies. In this experience have combined approaches that prioritize the use of ICT with other where evaluation becomes an element of learning. This has involved the use of virtual platforms to support teaching that facilitate learning and activities where no face-to-face are combined. The design of the components of the course is inspired by the dimensions proposed by Carless (2003) model. This model uses evaluation as a learning element. The development of this experience has shown how the didactic proposal has been positively interpreted by students. Students recognized that they had to learn and deeply understand the basic concepts of the subject, so that they can teach and assess their peers.

Keywords

Blended learning; Assessment; Higher education; Feedback
Aprendizaje Combinado; Evaluación; Educación Superior; Retroalimentación. 


\section{Introducción}

El Espacio Europeo de Educación Superior (EEES) establece como uno de sus principales objetivos el desarrollo de competencias personales y profesionales en el estudiante. Para ello, se precisa diseñar y desarrollar nuevas metodologías específicas para las asignaturas comprendidas en los planes de estudio, a la vez que se deben realizar investigaciones sobre la valoración de los estudiantes en torno a la calidad de la docencia universitaria (De-Juanas y Beltrán, 2013), y sobre los procedimientos de evaluación más adecuados y actuales. En las metodologías activas, el alumno debe ser el auténtico eje de la educación universitaria y el profesor un mediador del proceso de aprendizaje. Estas metodologías deben incorporar un justo grado de dificultad, para evitar que una carga cognitiva excesiva lleve a la pérdida de motivación hacia los estudios, ya que la motivación es condición fundamental del aprendizaje (López, Valentín y González-Tablas, 2012). Tampoco se debe olvidar, en relación con las condiciones del aprendiz, sus conocimientos previos y su predisposición hacia la comprensión, pues al enfrentase a una tarea extremadamente compleja el estudiante podría llegar a sentirse incapaz de finalizarla con éxito y por tanto, fracasar.

Las metodologías docentes empleadas actualmente en la Educación Universitaria aprovechan las innovaciones generadas por las TIC para pasar de un modelo de enseñanza tradicional a un nuevo modelo que combina la presencialidad clásica con la incorporación de variadas herramientas didácticas no presenciales, diversas tecnologías y metodologías de aprendizaje, o distintas localizaciones de espacios de aprendizaje (Cabero y Llorente, 2008). Dicho modelo de enseñanza ha recibido el nombre de blended learning o b-learning. Se han publicado numerosos trabajos sobre los beneficios que supone la aplicación de modelos b-learning. En ellos, se constatan las ventajas que encuentran los estudiantes, especialmente en lo referido al conocimiento sobre el trabajo que deben realizar, la información sobre los costes requeridos en cuanto al tiempo a emplear, la facilidad de acceso a los materiales y a los docentes, etc. (Cabero y Llorente, 2010).

Las metodologías activas de aprendizaje propician el trabajo colectivo, el intercambio de opiniones y la reflexión conjunta en torno a la experiencia de los actores involucrados (alumnos y profesores). De acuerdo con Vigotsky $(1979,1988)$, la interacción social juega un rol de vital importancia en el desarrollo cognitivo. Desde el constructivismo se sugiere la existencia de tres tipos de interacción en cualquier modalidad de enseñanza superior, sea esta presencial o no: la primera interacción se produce entre el estudiante y el contenido; la segunda, se refiere a la que establecen los estudiantes entre sí; $y$, la tercera se crea entre el tutor (ya sea el profesor o un compañero con formación previa en el contenido) y el estudiante. Junto con el tutor y los materiales, cualquiera que sea su naturaleza, 
la presencia de los iguales constituye también un recurso más para la progresión de su aprendizaje, teniendo un papel relevante en la eficacia de los procesos de adquisición de las competencias, tal como establece el aprendizaje colaborativo (Dillenbourg, 1999).

Por otro lado, tal y como plantean Spiro, Feltovich, Jacobson y Coulson (1992) en su teoría de la Flexibilidad Cognitiva, es necesario trabajar los conceptos en distintos con-textos para favorecer las representaciones múltiples de un mismo objeto, a fin de privilegiar la adquisición de conocimientos complejos y facilitar su transferencia. Es importante que el contexto donde se produce el aprendizaje sea auténtico y conectado con la realidad, ya sea de forma verdadera o simulada. Del mismo modo, se debe facilitar la ayuda, los recursos y las herramientas necesarias para que el aprendiz aborde gradual-mente los contenidos complejos. Es fundamental para el buen desarrollo de la autonomía y de la capacidad del estudiante, seleccionar metodologías activas y estrategias de aprendizaje que sean adecuadas a sus características, a sus estilos de aprendizaje y a la complejidad de los contenidos y objetivos de aprendizaje por alcanzar.

Tal como sugieren Quesada, Rodríguez-Gómez, e Ibarra (2013), los retos planteados en Europa con la construcción del EEES requieren buscar una metodología en la que la adquisición de competencias y la evaluación estén asociadas con el aprendizaje. La propuesta formulada por D. Carless (2003), la teoría LOA (Learning-Oriented Assessment) sobre la evaluación orientada al aprendizaje, aporta una notable mejora cualitativa a los procesos evaluativos en la educación superior, a la vez que busca coherencia con el entorno sociolaboral al que deben enfrentarse los nuevos egresados.

El contexto en el que se desarrolla este constructo hace hincapié en los aspectos de aprendizaje que conlleva el proceso evaluativo. La LOA se caracteriza como un indicador de las modalidades de evaluación en las que sobresalen más los elementos de aprendizaje que las mediciones (D. Carless, Joughin, y Liu, 2006; Carless, Joughin, y Mok, 2006). En la figura 1, se reproduce el modelo propuesto por Carless sobre la evaluación y el aprendizaje como una forma productiva de asociación (Carless, 2007), siendo los objetivos principales de la evaluación por una parte, el "elemento de la certificación" (objeto de certificación) que se centra principalmente en la evaluación de logros de los estudiantes, y por otra, el "elemento de aprendizaje".

La LOA representa un intento de reconciliar la evaluación formativa y la sumativa, a la vez que focaliza toda la evaluación en el desarrollo del aprendizaje de los estudiantes. Comprende tres dimensiones entrelazadas: 1) las tareas de evaluación como tareas de aprendizaje; 2) la participación de los estudiantes en la evaluación; y 3) la retroalimentación como alimentación directa.

Esta teoría promueve los aspectos de la evaluación que fomenten y fortalezcan el aprendizaje de los estudiantes. En la evaluación formativa el aprendizaje es una característica inherente y tiene como 
propósito verificar que el proceso de enseñanza-aprendizaje tuvo lugar (Gibbs y Simpson, 2004). La evaluación sumativa, según Knight (2002), consiste en la forma mediante la cual se mide y juzga el aprendizaje con el fin de certificarlo o asignar calificaciones. La intersección de los círculos en la Figura 1, indica que cuando la evaluación está funcionando de manera eficiente, no debería haber una superposición sustancial entre el "elemento de la certificación" y el "elemento de aprendizaje".
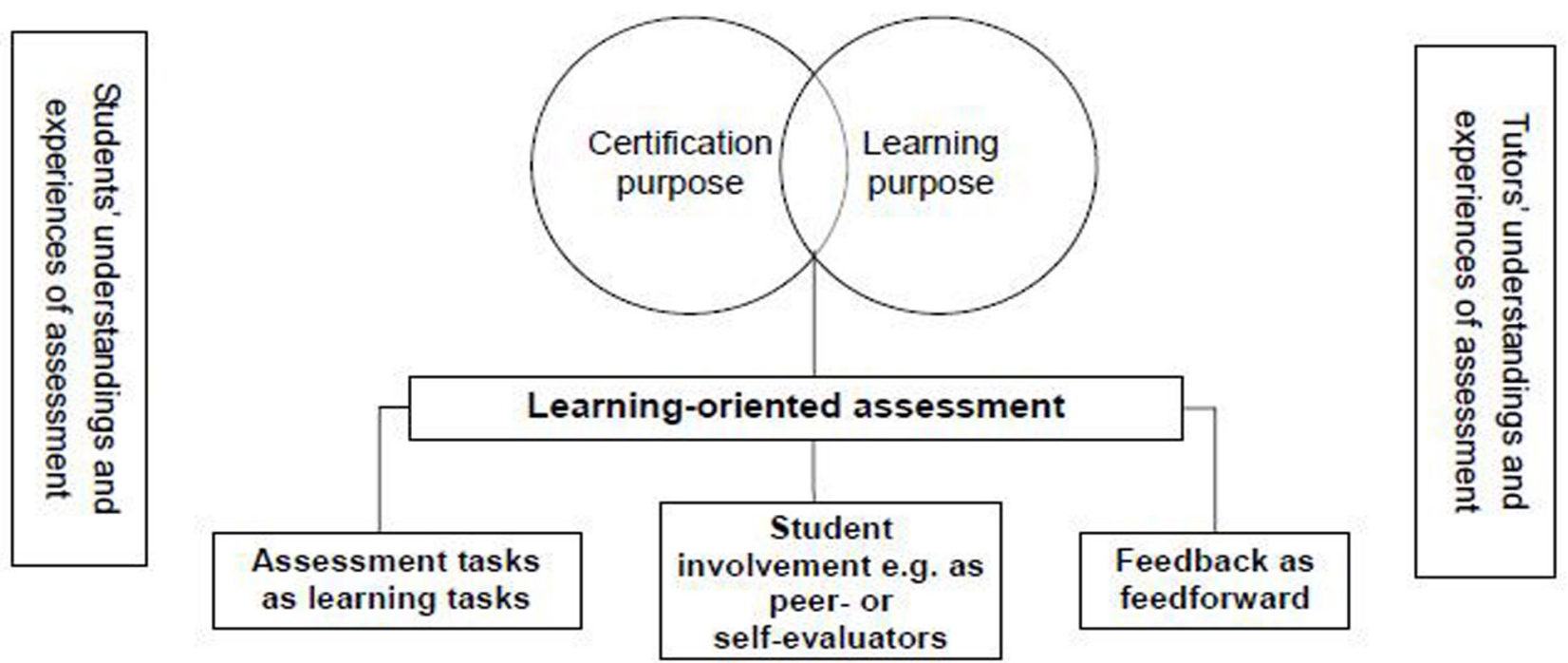

Figura 1. Marco teórico de la evaluación orientada al aprendizaje Fuente: Carless, D. (2007)

Resumiendo el esquema propuesto por Carless (2007), se pueden describir las dimensiones propuestas de la forma siguiente:

1. Las tareas de evaluación entendidas como tareas de aprendizaje. La evaluación debe promover el tipo de aprendizaje necesario para el futuro desempeño profesional de los estudiantes, ayudando a conseguir las metas a las que aspiran. Se precisa que incorpore los resultados del aprendizaje y que este sea en profundidad. Para ello, la función del docente consistirá en identificar, comprender y hacer corresponder entre sí, los objetivos con los contenidos de aprendizaje, con las actividades de enseñanza-aprendizaje y con la evaluación. Esta concepción está en sintonía con la teoría de la "alineación constructiva de la enseñanza" propuesta por J. Biggs al describirla como un sistema equilibrado, en el que todos sus elementos se interrelacionan formando un ecosistema (Biggs, 2006). Dicho conjunto de elementos configura un sistema interconectado dirigido a que el estudiante construya su propio aprendizaje y donde el profesor crea un entorno de tareas que lo propician (Hernández-Pina, Martínez, Da Fonseca, y Rubio, 2005). Cualquier desequilibrio en el sistema conduce al fracaso en el proceso de enseñanza, haciendo que el aprendizaje sea superficial, al estar más orientado al resultado de la evaluación que a la comprensión significativa de los conceptos.

2. La participación de los estudiantes como autoevaluadores y evaluadores de pares. El proceso de 
evaluación debe involucrar activamente a los estudiantes para promover el aprendizaje a lo largo de toda la vida. Por ello, debe promocionarse la autoevaluación y la evaluación entre compañeros, aunque esté plagada de tensiones y compromisos. Como Boud señaló, la evaluación tiene un carácter bifuncional en cada una de sus manifestaciones (Boud, 2000): trata sobre la clasificación y sobre el aprendizaje; sobre normas y comparaciones entre individuos; sobre la comunicación de mensajes explícitos e implícitos; y a la vez que es una cuestión técnica, impacta también sobre la vida emocional de los estudiantes. La evaluación debe estar basada en principios, pero también requiere práctica. Sus criterios deben ser públicos y alcanzados mediante el diálogo entre profesores y estudiantes. La evaluación así planteada debe realizar múltiples funciones, y la mejora de su puesta en práctica constituye todo un desafío, frente a la simplicidad de la evaluación tradicional.

3. La retroalimentación como alimentación directa (feedforward). La retroalimentación debe ser prospectiva, es decir, la información proporcionada por los tutores, sea un profesor o un igual, debe tener implicaciones para la tarea actual y para posibles tareas que el estudiante pueda realizar en el futuro. La retroalimentación debe estar dirigida al presente académico y al futuro profesional para mejorar la implicación y motivación del estudiante. Ello marca la diferencia con los planteamientos tradicionales en los que se prioriza que el docente solo busque justificar una valoración mediante una calificación (Sadler, 2002; Liu y Carless, 2006; Orsmond y Merry, 2011).

Estas tres dimensiones entrelazadas entre sí configuran un factor único en el que influyen las expectativas sobre la experiencia de evaluación que posean el tutor y los estudiantes (Figura 1). Es probable que la evaluación entre pares, o la autoevaluación, sea más eficaz cuando los estudiantes conocen y controlan los criterios y las normas de evaluación. Del mismo modo, la capacidad de los profesores para implementar la LOA puede verse limitada por su escasa experiencia en los diferentes tipos de metodologías activas de aprendizaje. Los estudiantes pueden ser reacios a aceptar los métodos de evaluación innovadores a menos que de ellos obtengan notables ventajas (Carless, 2007).

Desde este enfoque, se considera la evaluación como una poderosa herramienta para desarrollar competencias valiosas en los estudiantes, así como para mejorar su nivel de implicación y motivación (Ibarra, Rodríguez y Gómez, 2012). De forma semejante, algunos estudios muestran distintas relaciones entre el uso de las TIC (en particular las plataformas de apoyo a la docencia), la motivación y las estrategias de aprendizaje en alumnos de Educación Superior (Valentín et al., 2013). Se puede considerar que el uso específico de dichas plataformas representa una herramienta adecuada donde sustentar los diferentes contenidos y materiales educativos, e incorporar instrumentos de evaluación, todos ellos recursos necesarios para alcanzar los resultados de aprendizaje deseados.

Otra característica metodológica importante, consiste en entrenar al aprendiz en el papel de tutor, 
puesto que la mejor manera de aprender es enseñando. Cuando ponemos a un estudiante en situación de enseñar un determinado concepto a sus compañeros, le estamos ayudando a que se esfuerce en alcanzar competencias tanto específicas (derivadas del contenido que tiene que aprender) como de liderazgo, de control del grupo, y de reflexión. El ejercicio del rol de tutor requiere el dominio de métodos didácticos para la atención individualizada o de un grupo, así como ciertas facultades para resolver dudas académicas, orientar al aprendiz en metodologías y técnicas de estudio, diagnosticar las dificultades y realizar las acciones pertinentes para resolverlas, además de estimular el estudio independiente. Esta tarea permite al tutor experimentar prácticas muy enriquecedoras que se constituyen en un beneficio adicional, pues con ellas adquiere habilidades como la capacidad para distribuir el trabajo, compromiso y gestión de equipos humanos, o la planificación y evaluación de tareas, cualidades indispensables para el desempeño profesional.

\section{Objetivos}

Se propone el desarrollo de una experiencia didáctica en una asignatura de estadística del Grado de Psicología, donde se incorpora la evaluación como elemento de aprendizaje y se implica al estudiante como tutor de sus propios compañeros. Con esta experiencia se pretende motivar y estimular la implicación activa de los estudiantes, ya que los contenidos de la estadística tienden a ser considerados ajenos a su futuro desempeño profesional. Con ello se pretende estimular la participación de los alumnos en equipos de trabajo cooperativo, fomentar su participación dinámica, y favorecer la preparación comprensiva y progresiva de los contenidos de la asignatura, todo lo cual se debería reflejar en una mejora de las calificaciones académicas que ellos obtengan.

\section{Diseño de la asignatura de estadística aplicada a la psicología II}

\subsection{Descripción general de la asignatura}

La asignatura forma parte del Plan de estudios de Grado en Psicología de la Universidad de Salamanca. La impartición de la materia se desarrolla a través de clases presenciales y de la utilización de la plataforma de apoyo virtual a la docencia (Studium), en un contexto de blended learning. El participante típico es una alumna (82,17\%), entre 18 y 25 años, con competencias adquiridas en el uso de la plataforma educativa.

Desde el inicio del curso los estudiantes conocen los contenidos a impartir y las tareas que deben 
realizar. La calificación de la asignatura (Tabla 1) proviene de los diferentes indicadores determinados por las tareas que los aprendices deben realizar a lo largo del curso.

El diseño y desarrollo de esta asignatura se ha apoyado en diversos marcos de referencia, tal como se ha descrito anteriormente: del constructivismo se extrae el concepto de la cimentación del conocimiento en el proceso de reelaboración del contenido para transmitirlo a los compañeros, conforme a Vigotsky (1979, 1988); del aprendizaje colaborativo, la idea de que los estudiantes trabajen en grupos preparando el contenido de la materia para impartirlo posteriormente al resto de sus compañeros (Dillenbourg, 1999); E-learning, como parte relevante del proceso de enseñanzaaprendizaje que se canaliza a través de la plataforma virtual de formación, Studium, en el espacio creado al efecto; Evaluación orientada al aprendizaje (Carless, 2007; Carless et al., 2006; Carless, Salter, Yang, y Lam, 2011; McArthur y Huxham, 2013), en la que intervienen como actores los propios aprendices, asumiendo el papel de docentes de sus compañeros. Estos marcos teóricos, han formado parte del desarrollo de la metodología didáctica seguida en la asignatura durante los cursos 201 1/12, 2012/13, 2013/14, y 2014/15.

\subsection{Roles desempeñados por los agentes que intervienen en el proceso de enseñanza-aprendizaje}

El modelo que se presenta exige que, antes de informar sobre las tareas y la formación de grupos, se describan los diferentes papeles que van a desempeñar los estudiantes, así como la forma de valorar su desempeño. Los agentes implicados en el proceso de enseñanzaaprendizaje ejercen roles que son variables en función de la tarea que van a realizar. Así pues, el profesor deja de ser una figura central para convertirse en uno más de los actores, y protagonizar un papel de supervisor. Por su parte, el alumno se convierte en docente de sus compañeros, desplegando al mismo tiempo el papel de tutor y evaluador de los mismos. Además es, a su vez, evaluado y supervisado por el profesor, quien verifica si el estudiante ha adquirido el nivel de competencia necesario para ejercer como tutor no experto.

La figura 2 muestra los diferentes roles que desempeña el estudiante en función de la tarea de aprendizaje que desarrolla. Por ejemplo, cuando el estudiante desarrolla el trabajo de grupo, explicando el seminario práctico-presencial que le ha sido asignado, está ejerciendo el papel de profesor-tutor.

Las tareas se han diseñado para llevarse a cabo en grupos de diferente entidad, que se desarrollan en espacios físicos distintos. 


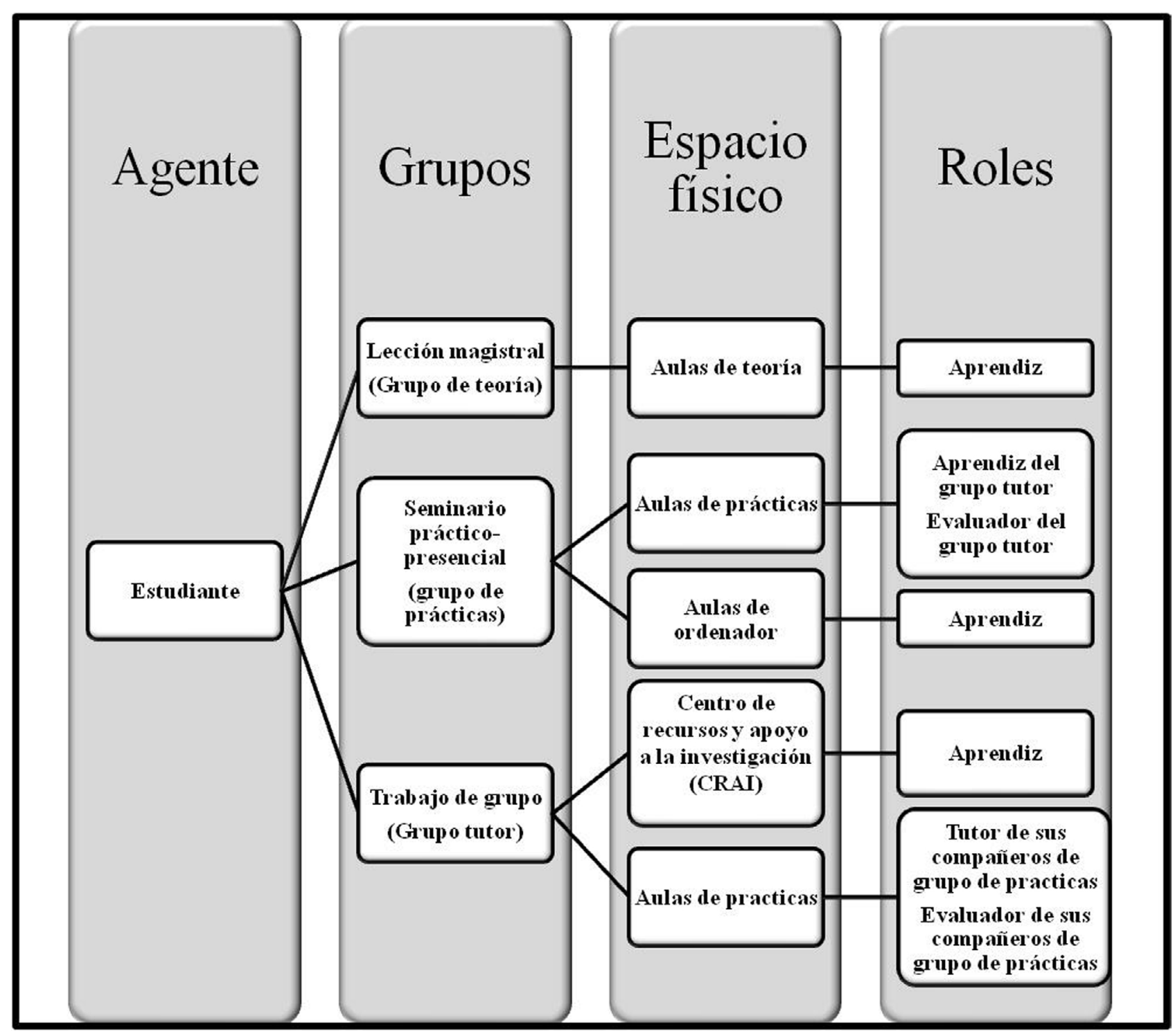

Figura 2. Roles del estudiante en función de los grupos de desempeño y el espacio físico donde se desarrolla la tarea

\subsection{Formación de grupos}

La formación de los grupos se ha realizado en función del número de alumnos matriculados en la asignatura y de la modalidad organizativa en la que está participando. Al ser estos más de 200 se les ha dividido en dos "grupos de teoría" (A y B), cada uno de los cuales se divide en tres "grupos de prácticas" (A1, A2, A3 y B1, B2, B3), con 36 alumnos aproximadamente. Estos grupos se dividen a su vez en 6 grupos de trabajo que cuentan con 5-7 participantes, denominándose "grupos tutores" (De A1.1 a B3.6).

\subsection{Diseño de tareas y la evaluación como elemento de aprendizaje}

En función de los contenidos de la materia, se diseñaron las diferentes modalidades organizativas en las que se articula el programa de enseñanza-aprendizaje que compone la asignatura. Se tuvo 
en cuenta la carga docente y el número de horas que el estudiante debe emplear en adquirir las competencias establecidas para la disciplina. Además se determinó el peso que corresponde a cada modalidad en la evaluación final. Estas modalidades organizativas son las siguientes:

$1^{\circ}$. Clases teóricas. Con ellas se pretende lograr un acercamiento a los contenidos sobre un tema, explicar procesos, efectuar demostraciones teóricas, presentar experiencias, etc., en definitiva, informar sobre los contenidos básicos de la materia. La exposición oral se apoya en recursos didácticos (audiovisuales, textos escritos, etc.) que facilitan la comunicación y permiten que los sujetos registren más información y activen más estrategias de aprendizaje. Para valorar esta modalidad se han utilizado dos estrategias distintas de evaluación: una dirigida al aprendizaje continuado, desarrollada on-line, que se ha denominado "prácticas en Studium"; y otra tradicional, que se corresponde con el "examen final" sobre los contenidos de toda la asignatura.

Con las prácticas en Studium se pretende priorizar tanto la retroalimentación dirigida al presente académico, como la dirigida al futuro profesional del estudiante. Para ello se han diseñado y desarrollado cuestionarios que incorporan casos prácticos muy relacionados con el desempeño laboral del psicólogo y ajustados a cada uno de los temas propuestos. Los cuestionarios se ponen a disposición del alumno vía on-line mediante la plataforma de apoyo a la docencia. Una vez que el cuestionario correspondiente ha sido resuelto y enviado, se proporciona la calificación del mismo (elemento de certificación), incorporando un feedback sobre cada una de las respuestas emitidas (elemento de aprendizaje).

$2^{\circ}$. Trabajo de grupo. Esta modalidad organizativa pretende alcanzar un doble objetivo. El primero consiste en estimular la participación de los alumnos en equipos de trabajo cooperativo fomentando su participación dinámica, donde todos los integrantes del equipo trabajen por igual. Con ello se pretende favorecer la preparación comprensiva y progresiva de los contenidos de la asignatura, bajo el principio de "aprender enseñando". Como segundo objetivo se pretende entrenar al aprendiz en el papel de docente, involucrándole activamente en las tareas de tutorización y evaluación. Tal proceso de evaluación implica una retroalimentación en tiempo real del desempeño de los compañeros, promoviendo su aprendizaje. Para ello, al comienzo del curso se distribuye a los alumnos en los denominados grupos tutores, los estudiantes que los integran deben elaborar, explicar, tutorizar y evaluar dos seminarios que les han sido asignados aleatoriamente de los doce que componen los seminarios práctico-presenciales.

El trabajo en grupo proporciona al estudiante un conocimiento de la asignatura de carácter global y tiene un potencial explicativo que le sitúa en condiciones de aprendizaje más significativas. La tarea del estudiante en su papel de tutor, le permite experimentar prácticas muy enriquecedoras. Estas proporcionan un beneficio adicional, pues con ellas el alumno adquiere otras habilidades 
indispensables para el desempeño profesional, como el liderazgo, la capacidad para distribuir el trabajo, el compromiso y gestión de equipos, o la planificación y evaluación de tareas.

El trabajo de grupo está orientado y guiado en todo momento por el profesor mediante la realización de, al menos, dos tutorías de carácter obligatorio. Cada tutoría se realiza con más de una semana de antelación a la impartición del seminario práctico-presencial y está convocada desde comienzo de curso en el organigrama semanal de la asignatura. En ellas se expone y discute sobre la forma de presentación, así como sobre los criterios que se deben seguir para la evaluación de la tarea que explicarán al resto de sus compañeros; se resuelven dudas, se corrigen los ejercicios y se proponen otros nuevos. Además le sirve al profesor para ir evaluando de forma continuada la evolución del trabajo.

Al finalizar el curso, cada grupo elabora una memoria con formato portafolio, señalando los puntos fuertes y débiles respecto a las tareas realizadas como grupo tutor. Además incorpora una valoración individual de los componentes del equipo a lo largo del curso. La memoria debe subirse a la plataforma Studium antes de defenderla oralmente en sesión pública y en la fecha prevista en el calendario.

$3^{\circ}$. Seminarios práctico-presenciales. Se fundamentan en actividades prácticas de papel y lápiz para desarrollar aprendizajes activos a través de la solución de problemas, aplicando las habilidades y conocimientos adquiridos. Dichas prácticas son impartidas por el grupo tutor (Ej.: A1.1) al grupo de prácticas (Ej.: A1) y supervisadas por el profesor. Se han diseñado 12 seminarios práctico-presenciales de los cuales cada alumno recibe 10 e imparte 2.

Cada seminario práctico-presencial tiene una duración de dos horas, estructurada en cuatro fases:

$1^{\text {a }}$ Fase: el grupo tutor expone ante su grupo de prácticas los puntos más relevantes de los contenidos teóricos de la práctica que les haya correspondido. Seguidamente, han de presentar el procedimiento a seguir para resolver un problema-tipo vinculado con los contenidos anteriores.

$2^{a}$ Fase: propone a su grupo de prácticas un ejercicio para su resolución en grupos pequeños apoyados por un miembro del equipo.

$3^{a}$ Fase: el grupo tutor evalúa a su grupo de prácticas mediante unos ejercicios que han creado específicamente para el seminario, calificando las respuestas de cada estudiante en una escala de 0 a 10.

$4^{\text {a }}$ Fase: en los últimos cinco minutos del seminario práctico-presencial se pide al grupo de prácticas que cumplimenten una encuesta de calidad docente, evaluando el trabajo realizado por el grupo tutor. Esta encuesta basada en rúbricas consta de cinco ítems sobre el desempeño del tutor (proporciona explicaciones, estimula el aprendizaje, anima a participar, etc.) y dos ítems sobre la eficacia de la práctica para ayudar al estudiante a fijar contenidos. 
Se han proyectado para ser realizados en grupo y de forma individual, produciéndose los tres tipos de interacción que desde el constructivismo social se sugieren: el estudiante con la asignatura, los estudiantes entre sí, y la tercera, la que se establece entre el estudiante y su grupo tutor. La interacción facilita un trabajo que siendo primero colaborativo, deriva en un trabajo individual en el que el alumno es capaz de resolver sus ejercicios. La evaluación de esta tarea es realizada por el grupo tutor de cada práctica, y está orientada al aprendizaje siguiendo el modelo de la teoría LOA. Tal evaluación parte del conocimiento cercano que tiene el estudiante, en su papel de docente, para identificar, comprender y hacer comprensivos los contenidos del aprendizaje y las actividades de enseñanza-aprendizaje que se precisan.

$4^{\circ}$. Seminarios de ordenador. Son actividades prácticas informatizadas mediante el uso de software estadístico, para desarrollar igualmente aprendizajes activos a través de la resolución de problemas. Como en la modalidad organizativa anterior, se pueden realizar bien en grupos de trabajo, bien de forma individual. Los alumnos de un mismo grupo trabajan colaborativamente, pero cada alumno individualmente aporta sus resultados en un cuaderno de prácticas. A diferencia de los seminarios práctico-presenciales, la evaluación de los contenidos de aprendizaje la realiza exclusivamente el profesor.

\subsection{Tareas, agentes, productos y elementos de certificación}

El desarrollo de las modalidades organizativas se ha vertebrado atendiendo a las diferentes tareas, agentes, productos de aprendizaje y elementos de certificación que componen la secuencia didáctica de la asignatura (Tabla 1). Dependiendo del tipo de tarea, el agente que la lleva a cabo es distinto. Asimismo cambia el agente evaluado, así como quien evalúa su desempeño. Además, los productos esperados, los instrumentos de evaluación y la certificación son específicos para cada una de las tareas a realizar. Desde el inicio del periodo lectivo, todos los estudiantes matriculados tienen acceso en Studium a los bloques de contenido y el desarrollo de cada uno de sus temas, junto con la información referida a los criterios de certificación, horarios, fechas de tutorías, asignación a los diferentes grupos y a los seminarios práctico-presenciales, etc.

Las estrategias e instrumentos de evaluación para la acreditación de los estudiantes se llevaron a cabo de forma continuada durante el curso, combinándose los siguientes tipos de pruebas y tareas realizadas:

a) Prácticas de Studium: constan de 11 cuestionarios (uno por tema), con formato similar al establecido para el examen final. Cada uno de los cuestionarios se valora de 0 a 10 y es necesario que al menos el 80\% tenga una calificación superior a 5 para que puedan ser tenidos en cuenta en la evaluación final. 


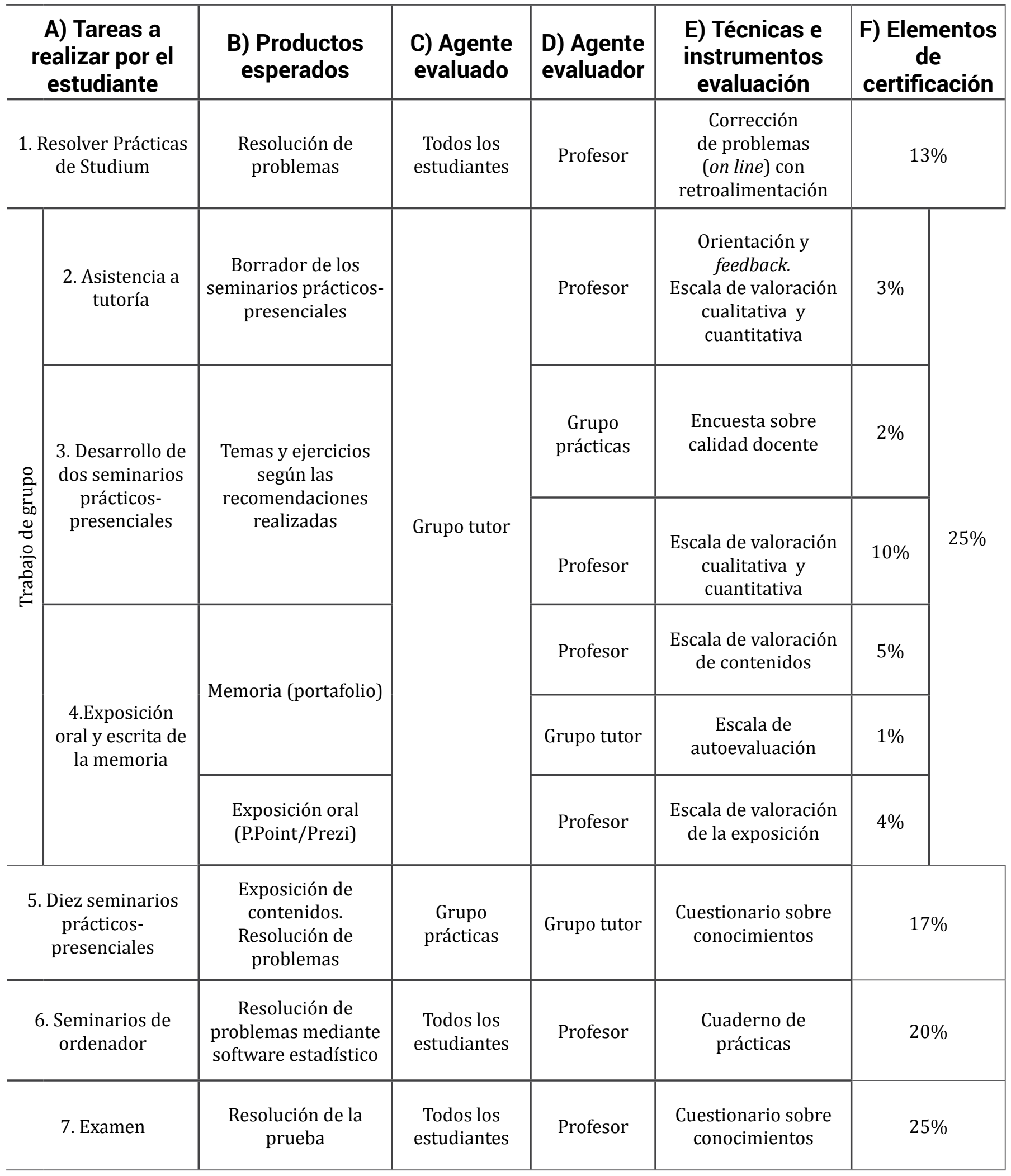

Tabla 1. Vinculación entre tareas, agentes, productos de aprendizaje y elementos de certificación

b) Trabajo de grupo: para evaluar este trabajo será necesario asistir obligatoriamente a dos sesiones de tutoría en grupo (Tabla 1, A-2), para el control del mismo. El docente tiene en cuenta para la certificación de esta tarea las asistencias obligatorias y la calificación numérica obtenida (Tabla 1, F-2). 
c) Seminarios práctico-presenciales y de ordenador: los alumnos, individualmente, tienen que entregar las actividades prácticas que se realicen en clase (Tabla 1, A-3, A-4 y A-5) y en el laboratorio de informática (Tabla 1, A-6) a lo largo del curso y en las fechas programadas. La asistencia a clase es obligatoria para poder participar en este tipo de actividad y será obligatorio entregar, como mínimo, el 80\% del total de las prácticas para que puedan añadirse a la calificación final.

d) Examen: consta de una parte teórica y otra práctica (Tabla 1, A-7). En la parte teórica se evalúa la comprensión de los conceptos más importantes de la materia, a través de una prueba objetiva con alternativas de respuesta. En la parte práctica se evalúa la habilidad para seleccionar, aplicar e interpretar los conceptos y las técnicas estadísticas que se imparten en la asignatura.

Para esta experiencia, se han generado, entre otros, diversos instrumentos evaluadores del papel del aprendiz como tutor no experto (Varela, Giralt, y Gallego, 2012). Para ello se han utilizado los criterios evaluadores de Dolmans y Ginns, (2005) que han mostrado buena fiabilidad y validez en población universitaria. En esta experiencia, los cinco factores extraídos por Dolmans y Ginns se han reducido a una pregunta por factor.

\section{Resultados académicos de la experiencia}

El presente estudio se basa en el análisis de los resultados académicos obtenidos por los estudiantes desde el curso 2007/08 a 2014/15 en la asignatura Estadística Aplicada a la Psicología II. Durante los cursos 2007/2008 a 2010/2011 la asignatura se impartió dentro del plan de estudios de la Licenciatura en Psicología, mientras que en los cursos restantes la asignatura fue impartida en la nueva titulación del Grado en Psicología. Por otra parte, durante los cursos 2009/10 y 2010/11 se incorporaron recursos y tareas (Plataforma Studium, cuestionarios on line y un trabajo de grupo) dirigidas a los estudiantes, que no siendo de carácter obligatorio, sin embargo contribuían a incrementar la calificación obtenida. En los cursos posteriores, hasta la actualidad, la asignatura ha sido impartida siguiendo el modelo didáctico propuesto en este trabajo.

Se llevó a cabo un estudio retrospectivo de los resultados académicos obtenidos por los estudiantes matriculados en la asignatura. Cuando se tiene en cuenta el total de los alumnos matriculados, el porcentaje de alumnos calificados como No presentado varía en función del curso académico (Tabla 2).

Así pues, en los cursos correspondientes a la Licenciatura (2007/08 a 2010/2011), los estudiantes no presentados constituyeron la categoría más frecuente (38,3\%, 50,2\%, 48,3\% y 21,9\% respectivamente). Por otra parte, en los cursos correspondientes a la Licenciatura el porcentaje de estudiantes con la 


\section{CALIFICACIONES}

\begin{tabular}{cccccccc}
\hline \multirow{2}{*}{ Curso } & & $\begin{array}{c}\text { No } \\
\text { Presentado }\end{array}$ & Suspenso & Aprobado & Notable & Sobresaliente & TOTAL \\
\hline 2008 & $\mathrm{n}$ & 173 & 161 & 85 & 29 & 4 & 452 \\
& $\%$ & $38,3 \%$ & $35,6 \%$ & $18,8 \%$ & $6,4 \%$ & $0,9 \%$ & $100,0 \%$ \\
\hline 2009 & $\mathrm{n}$ & 206 & 106 & 55 & 39 & 4 & 410 \\
& $\%$ & $50,2 \%$ & $25,9 \%$ & $13,4 \%$ & $9,5 \%$ & $1 \%$ & $100,0 \%$ \\
\hline \multirow{2}{*}{2010} & $\mathrm{n}$ & 186 & 71 & 87 & 38 & 3 & 385 \\
& $\%$ & $48,3 \%$ & $18,4 \%$ & $22,6 \%$ & $9,9 \%$ & $0,8 \%$ & $100,0 \%$ \\
\hline 2011 & $\mathrm{n}$ & 92 & 79 & 207 & 40 & 2 & 420 \\
& $\%$ & $21,9 \%$ & $18,8 \%$ & $49,3 \%$ & $9,5 \%$ & $0,4 \%$ & $100,0 \%$ \\
\hline 2012 & $\mathrm{n}$ & 2 & 19 & 152 & 9 & 0 & 182 \\
& $\%$ & $1,1 \%$ & $10,4 \%$ & $83,5 \%$ & $4,9 \%$ & $0,0 \%$ & $100,0 \%$ \\
\hline \multirow{2}{*}{2013} & $\mathrm{n}$ & 7 & 14 & 190 & 4 & 0 & 215 \\
& $\%$ & $3,3 \%$ & $6,5 \%$ & $88,4 \%$ & $1,9 \%$ & $0,0 \%$ & $100,0 \%$ \\
\hline 2014 & $\mathrm{n}$ & 3 & 21 & 184 & 11 & 0 & 219 \\
& $\%$ & $1,4 \%$ & $9,6 \%$ & $84,0 \%$ & $5,0 \%$ & $0,0 \%$ & $100,0 \%$ \\
\hline 2015 & $\mathrm{n}$ & 5 & 24 & 178 & 9 & 0 & 216 \\
& $\%$ & $2,3 \%$ & $11,1 \%$ & $82,4 \%$ & $4,2 \%$ & $0,0 \%$ & $100,0 \%$ \\
\hline \multirow{2}{*}{ Total } & $\mathrm{n}$ & 674 & 495 & 1138 & 179 & 13 & 2499 \\
& $\% *$ & $27 \%$ & $19,8 \%$ & $45,5 \%$ & $7,2 \%$ & $0,6 \%$ & $100 \%$ \\
\hline \multirow{2}{*}{20} & & & & & \\
\end{tabular}

$\% *$ Dentro del curso académico

Tabla 2. Distribución de las calificaciones en función del curso académico.

calificación Sobresaliente, ha sido 0,9\%, 1\%, 0,8\% y 0,4\%, respectivamente. Ello es particularmente relevante dado que desde la implantación del Grado no ha habido ningún estudiante con dicha calificación. Sin embargo, el porcentaje de estudiantes con la calificación Aprobado, se ha incrementado muy considerablemente a lo largo de los cursos correspondientes al Grado y, en consecuencia, el porcentaje de estudiantes con la calificación Suspenso, se ha reducido drásticamente.

De los 2499 estudiantes matriculados, solo 1823 constan en actas con una nota cuantitativa entre 0 y 100, mostrándose sus valores descriptivos en la tabla 3.

A partir de los datos obtenidos, se llevó a cabo un ANOVA unifactorial, utilizando el curso académico como factor inter y las puntuaciones obtenidas como variable de medida, a un nivel de confianza del 95\%. Los resultados indican la existencia de efectos debidos al factor $F(1,1815)=36,45, p<$ ,000. La prueba a posteriori DHS de Tukey mostró la existencia de tres subconjuntos homogéneos de calificaciones. El primero de ellos está formado por las calificaciones de los cursos 2007 a 
2009, agrupando a las puntuaciones más bajas $(45,00)$. El segundo subconjunto lo configuran las calificaciones de los cursos 2009 a 2011, recogiendo las puntuaciones intermedias (53,78). El último subconjunto está formado por los cursos restantes (2012 a 2015), agrupando las calificación medias más elevadas $(57,79)$. Dichos subconjuntos se corresponden con los periodos antes mencionados respecto a los planes de estudio de la Licenciatura y el Grado, así como el periodo de transición en donde se incorporaron metodologías y recursos propios del blended learning.

\begin{tabular}{cccc} 
Curso Académico & Nota Media & Desviación típica & N \\
\hline $2007 / 08$ & 43,06 & 20,02 & 277 \\
$2008 / 09$ & 46,95 & 22,80 & 204 \\
$2009 / 10$ & 53,91 & 18,65 & 199 \\
$2010 / 11$ & 53,64 & 13,19 & 328 \\
$2011 / 12$ & 58,32 & 8,04 & 180 \\
$2012 / 13$ & 58,61 & 6,42 & 208 \\
$2013 / 14$ & 56,55 & 8,88 & 216 \\
$2014 / 15$ & 58,49 & 9,12 & 211 \\
\hline Total & 53,25 & 15,75 & 1823 \\
\hline
\end{tabular}

Tabla 3. Datos descriptivos de la muestra en función del curso académico

\section{Valoración de la experiencia: fortalezas y debilidades}

Tradicionalmente, en la universidad se han valorado sobre todo las exposiciones teóricas y las clases magistrales que llevan a adquirir un conocimiento teórico de las materias que se imparten, haciendo especial hincapié en aspectos memorísticos y de pensamiento reproductivo, confeccionando una evaluación de carácter eminentemente sumativo, bien porque se fundamentara en la realización de uno o más exámenes, bien porque exclusivamente se evaluaban conocimientos.

Con la incorporación al modelo Bolonia, la universidad ha experimentado cambios sustanciales en sus planteamientos, destacando entre ellos la implementación de metodologías blended learning en el proceso de enseñanza-aprendizaje. En este marco, la clase magistral deja de ser el centro de la docencia, y el profesor pierde su carácter exclusivo de transmisor de conocimientos, para convertirse en un apoyo continuado a lo largo de los estudios universitarios del estudiante. En la actualidad, el estudiante es el protagonista fundamental de su aprendizaje, no solo en los aspectos directamente relacionados con su objeto de estudio, sino también con aquellas competencias que le van a permitir integrarse de forma progresiva al mundo profesional. 
Al mismo tiempo, otro de los cambios importantes ha consistido en la nueva concepción de la evaluación, al modificarse tanto el objeto como el procedimiento de la misma. Así el objeto de evaluación ya no son estrictamente los contenidos de la materia adquiridos por el estudiante, sino las competencias alcanzadas. De igual modo, no es el profesor el único agente evaluador, y los instrumentos de evaluación inciden tanto en aspectos cuantitativos como cualitativos. En este sentido, se ha pasado de una evaluación puramente acreditativa a modelos tan diversos como el propuesto por Carless et al. (2006), donde la evaluación y el aprendizaje se funden en una forma productiva de asociación.

El diseño de la asignatura de Estadística Aplicada a la Psicología II, descrito en el presente trabajo, se ha basado en el modelo LOA, de tal manera que las dimensiones del modelo se ven reflejadas en el desarrollo de las tareas descritas anteriormente.

La primera dimensión de este marco teórico, entiende las tareas de evaluación como tareas de aprendizaje. Todas las tareas de la asignatura han sido diseñadas para alcanzar el objetivo que persigue esta dimensión, permitiendo al estudiante construir su propio aprendizaje sobre la estadística. Sin embargo, se ha podido detectar, conforme a los resultados reflejados en la Tabla 2, que con este modelo de enseñanza es más difícil reconocer la calidad superior de algunos estudiantes, porque se homogenizan y diluyen las competencias extraordinarias que algunos de ellos puedan adquirir, dado que en trabajos de equipo y tareas no individualizadas, tales competencias son difíciles de detectar, y, por tanto, no pueden ser evaluadas y reconocidas.

La siguiente dimensión, relacionada especialmente con la participación de los alumnos en las tareas de evaluación, siguiendo a Boud y Falchikov (2006), fue consensuada entre el profesor y los estudiantes al inicio del curso académico, especialmente en aquellas tareas que se realizaron en grupo y en los seminarios. El hacer un seguimiento continuado y estimulado por los compañeros, favoreció que todos participaran y se integraran en los grupos. Estos, a veces se han relacionado entre sí de forma competitiva, cuestión esta que en principio pudiera considerarse como una consecuencia beneficiosa, pero en otras ocasiones ha supuesto un perjuicio para aquellos otros grupos que eran más asociativos. Además la evaluación que realizaron de sus compañeros, en algunos casos, fue más estricta que la realizada por el propio profesor, en contra de lo pudiera esperarse. Por otro lado, la escasa diferenciación presente en las calificaciones académicas durante los cursos académicos correspondientes al Grado, también puede ser debida a que parte de la evaluación es realizada por los propios compañeros del estudiante, y esta suele ser homogénea para todos los miembros del grupo, desapareciendo así las posibles diferencias que pudieran existir.

La tercera dimensión refleja la retroalimentación, proporcionada tanto por el tutor como por los compañeros. A lo largo de esta experiencia, hemos podido detectar que los alumnos no percibieron 
el contexto didáctico de las tareas del mismo modo que el profesor. La información procedente de la retroalimentación era valorada de forma más satisfactoria cuando la fuente de la misma eran los iguales y, por otro lado, los alumnos demandaban una retroalimentación más inmediata y frecuente en el diálogo que se establecía con el profesor. Tal y como apuntan McArthur y Huxham, (2013), a la hora de introducir el feedback, tuvieron especial importancia la claridad, el control y la oportunidad del momento, porque se precisaron para que el estudiante pudiera construir su particular concepción de la retroalimentación, al compartirlos con el docente cuando actuaron como tutores. En consecuencia, tal y como apuntan Carless et al. (2011), la retroalimentación fue sostenible y acertada, cuando apoyó a los estudiantes en el autocontrol de su propio trabajo, de forma independiente del profesor, hecho puesto de manifiesto por nuestros estudiantes en las memorias presentadas durante los cursos académicos correspondientes al Grado.

En esta experiencia docente, considerada una aplicación del blended learning en cuanto al moderado uso de los componentes online (Cabero y Llorente, 2008), la plataforma de apoyo a la docencia ha sido un instrumento de vertebración de las interacciones entre los diferentes componentes del entorno educativo. Ha permitido al aprendiz, desde el inicio, hacerse una idea general del curso, organizar su trabajo y facilitarle el acceso rápido a los materiales necesarios para cada una de las tareas diseñadas. Además, el uso de los foros creados en la plataforma, permitió a los estudiantes la interacción con sus compañeros de grupo, compartir sus materiales de trabajo, contactar con el profesor, etc. De este modo, la plataforma ha contribuido de manera sustancial a entender mejor la estructura y dinámica interna de la asignatura.

En cuanto a la tutorización por pares, basándonos en las manifestaciones expresadas en las memorias de trabajo elaboradas por los estudiantes, ha resultado ser una estrategia especialmente beneficiosa: en general todos los grupos han afirmado que entendieron muchos de los contenidos de la asignatura cuando tuvieron que explicárselos a sus compañeros. Bajo la utilización que hemos realizado de la LOA en este trabajo se supera el concepto alumno-tutor (Cieza, 2011), introduciendo la figura del alumno-tutor-evaluador. Los alumnos manifestaron que la evaluación realizada a sus compañeros les supuso un gran esfuerzo y responsabilidad, criticando incluso a aquellos de sus compañeros que adoptaban una valoración común para todos los miembros del equipo, evitando así el compromiso que conlleva la realización del proceso evaluador. Además, afırmaron comprender mejor la tarea del docente. De aquí, se deriva también que la estrategia de evaluación seguida en la asignatura, ha cumplido el objetivo que se perseguía debido a que, tal como los estudiantes afirmaron, para evaluar necesitan conocer. No obstante, recordemos que las aportaciones de los estudiantes revelaron cómo la retroalimentación realizada por el profesor, debería llevarse a cabo con mayor frecuencia. Dicha retroalimentación requeriría contar con una plantilla de profesorado mayor de la disponible en la actualidad o reducir el número de alumnos. 
Las valoraciones de los estudiantes inciden en aspectos educativos ligeramente alejados del blended learning, ya que como mejora del curso solo han demandado una mayor cantidad de tutorías presenciales. Por otro lado, respecto al uso de foros, los estudiantes manifestaron mayoritariamente preferir sistemas de mensajería multimedia (WhatsApp, Line, etc.), ajenas a las herramientas de comunicación proporcionadas por las plataformas de apoyo a la docencia (Moodle).

Desde un punto de vista cuantitativo, el análisis de los resultados de las diferentes modalidades e instrumentos de evaluación aplicados, ha permitido establecer y delimitar objetivamente las bondades (aumento de número de estudiante presentados y aprobados) y limitaciones (discriminar positivamente a aquellos que han conseguido un mayor grado de competencias en la asignatura) del modelo utilizado.

Además, hemos comprobado cómo se produce un primer cambio en los dos últimos cursos académicos correspondientes a la Licenciatura (que previamente hemos considerado como período de transición), en los cuales se incorporaron elementos característicos del blended learning, tales como la plataforma de apoyo virtual a la docencia, en su doble vertiente de herramienta facilitadora de recursos educativos y como medio para la presentación de instrumentos de evaluación con retroalimentación inmediata. El segundo cambio ha coincidido con el inicio del Grado, iniciándose entonces la implantación completa del modelo. Los resultados relativos a este periodo ponen de manifiesto la existencia de un incremento en la calificación media, unida a una gran disminución de la variabilidad de las notas obtenidas. Por último, se aprecia un cambio cualitativo importante en cuanto a la drástica disminución del número de abandonos (no presentados) y el aumento simultáneo de la implicación y seguimiento por parte de los estudiantes matriculados en el curso.

En definitiva, se ha intentado que los alumnos desplegasen habilidades de pensamiento e instrumentales que contribuyesen al desarrollo de destrezas metacognitivas para el aprendizaje, a través de la realización de las tareas académicas diseñadas en el contexto de la asignatura. Con ello se aspira a que estas destrezas puedan generalizarse al futuro profesional y personal del aprendiz y además le permitan adaptarse a situaciones de diversa índole y entidad.

\section{Agradecimientos}

Agradecimientos a la Universidad de Salamanca por la concesión de la subvención recibida para la realización del proyecto (ID2012/300) en la convocatoria de Ayudas a Proyectos de Innovación Didáctica 2012/2013. 


\section{Referencias}

Biggs, J. (2006). Calidad en el aprendizaje universitario. Madrid: Narcea Ediciones.

Boud, D. (2000). Sustainable assessment: rethinking assessment for the learning society. Studies in Continuing Education, 22(2), 151-167. doi:http://dx.doi.org/10.1080/713695728

Boud, D., \& Falchikov, N. (2006). Aligning assessment with long-term learning. Assessment \& Evaluation in Higher Education, 37(4), 399-413. doi:http://dx.doi.org/10.1080/02602930600679050

Cabero, J., \& Llorente, M. C. (2008). Del eLearning al Blended Learning: nuevas acciones educativas. Quaderns Digitals, 51 (ISSN 1575-93939). Recuperado el 20 de julio de 2014, de: http://tecnologiaedu. us.es/cuestionario/bibliovir/jca19.pdf.

Cabero, J., \& Llorente, M. C. (2010). La experiencia formativa de los alumnos en el Campus Andaluz Virtual (CAV). RU\&SC. Revista de Universidad y Sociedad del Conocimiento, 7, (2)1-14.

Carless, D. (2003). Factors in the implementation of task-based teaching in primary schools. System, 37(4), 485-500. doi:http://dx.doi.org/10.1016/j.system.2003.03.002.

Carless, D. (2007). Learning-oriented assessment: Conceptual bases and practical implications. Innovations in Education and Teaching International, 44(1), 57-66. doi:http://dx.doi. org/10.1080/14703290601081332

Carless, D., Joughin, G., \& Liu, N. F. (2006). How assessment supports learning: Learning-oriented assessment in action. Hong Kong: Hong Kong University Press. doi:http://dx.doi.org/10.5790/ hongkong/9789622098237.001.0001

Carless, D., Joughin, G., \& Mok, M. M. C. (2006). Learning-oriented assessment: principles and practice. Assessment \& Evaluation in Higher Education, 37(4), 395-398. doi:http://dx.doi. org/10.1080/02602930600679043

Carless, D., Salter, D., Yang, M., \& Lam, J. (2011). Developing sustainable feedback practices. Studies in Higher Education, 36(4), 395-407. doi: http://dx.doi.org/10.1080/03075071003642449. 
Cieza, J. A. (2011). El proyecto «tutoría entre compañeros» en la Facultad de Educación de la Universidad de Salamanca. Aula, 17, 249-254.

De-Juanas Oliva, Á., \& Beltrán Llera, J. A. (2013). Valoraciones de los estudiantes de ciencias de la educación sobre la calidad de la docencia universitaria. Educación XX1, 17(1). Recuperado a partir de http://e-spacio.uned.es/revistasuned/index.php/educacionXX1/article/view/10705.

Dillenbourg, P. (1999). What do you mean by collaborative learning? En Collaborative-learning: Cognitive and computational approaches. (pp. 1-19). Oxford: Elsevier. Recuperado a partir de http://halshs. archives-ouvertes.fr/docs/00/19/02/40/PDF/Dillenbourg-Pierre-1999.pdf.

Dolmans, D. H. J. M., \& Ginns, P. (2005). A short questionnaire to evaluate the effectiveness of tutors in PBL: validity and reliability. Medical Teacher, 27(6), 534-538. doi: http://dx.doi. org/10.1080/01421590500136477.

Gibbs, G., \& Simpson, C. (2004). Conditions under which assessment supports students' learning. Learning and Teaching in Higher Education, 1(1), 3-31.

Hernández-Pina, F., Martínez, P., Da Fonseca, P., \& Rubio, M. (2005). Aprendizaje, competencias y rendimiento en Educación Superior. Madrid: Editorial La Muralla.

Ibarra Sáiz, M. S., Rodríguez Gómez, G., \& Gómez Ruiz, M. Á. (2012). La evaluación entre iguales: beneficios y estrategias para su práctica en la universidad. Revista de Educación, 359, 206-231. doi: http://dx.doi.org/10-4438/1988-592X-RE-2010-359-092.

Knight, P. T. (2002). Summative Assessment in Higher Education: Practices in disarray. Studies in Higher Education, 27(3), 275-286. doi: http://dx.doi.org/10.1080/03075070220000662

Liu, N. F. y Carless, D. (2006). Peer feedback: the learning element of peer assessment. Teaching in Higher Education, 17(3), 279-290. http://dx.doi.org/10.1080/13562510600680582

López, E., Valentín, A., \& González-Tablas, M. M. (2012). De las condiciones del prácticum de la licenciatura en psicología a las competencias del grado: análisis de las valoraciones de los alumnos. En González Ferreras, C., González Manjón, D., Mestre Navas, J. M., \& Guil Bozal, R. (Coords.), Aportaciones recientes al estudio de la Motivación y las Emociones (pp. 47-55). Cádiz: Fénix Editora. 
McArthur, J., \& Huxham, M. (2013). Feedback unbound: From master to usher. En S. Merry, M. Price,

D. Carless y M. Taras, Eds. Reconceptualising Feedback in Higher Education: Developing dialogue with students. London: Routledge.

Orsmond, P., \& Merry, S. (2011). Feedback alignment: effective and ineffective links between tutors' and students' understanding of coursework feedback. Assessment \& Evaluation in Higher Education, 36(2), 125-136. http://dx.doi.org/10.1080/02602930903201651

Quesada Serra, V., Rodríguez-Gómez, G., \& Ibarra Sáiz, M. S. (2013). ActEval: un instrumento para el análisis y reflexión sobre la actividad evaluadora del profesorado universitario. Revista de Educación, (362. Septiembre - Diciembre), 69- 104. doi: http://dx.doi.org/10.4438/1988-592X-RE-2011-362-153.

Sadler, D. R. (1989). Formative assessment and the design of instructional systems. Instructional science, 18(2), 119-144. http://dx.doi.org/10.1007/BF00117714

Sadler, D. R. (2002). Learning dispositions: can we really assess them? Assessment in Education: Principles, Policy \& Practice, 9(1), 45-51.

Spiro, R. J., Feltovich, P. J., Jacobson, M. J. y Coulson, R. L. (1992). Cognitive flexibility, constructivism, and hypertext: random access instruction for advanced knowledge acquisition in ill-structured domain. En Constructivism and the technology of instruction: a conversation. Hillside, NJ: Lawrence Erlbaum Associates.

Valentín, A., Mateos, P. M., González-Tablas, M. M., Pérez, L., López, E., \& García, I. (2013). Motivation and learning strategies in the use of ICTs among university students. Computers \& Education, 67, 5258. http://dx.doi.org/10.1016/j.compedu.2012.09.008

Varela, J. L. M., Giralt, E. G., \& Gallego, G. A. (2012). El ABP en los estudios universitarios de las artes. Un análisis del papel del tutor experto y no-experto en la identificación de los objetivos de aprendizaje. CIDUI-Llibre d'actes, 7(1). Recuperado a partir de http://www.cidui.org/revista-cidui12/index.php/ cidui12/article/view/53.

Vigotsky, L. S. (1979). El desarrollo de los procesos psíquicos superiores. Barcelona: Editorial Crítica.

Vigotsky, L. S. (1988). Interacción entre enseñanza y desarrollo. En Selección de lecturas de Psicología Pedagógica y de las Edades, Tomo III. La Habana: Editora Universidad. 